\title{
Environmental Monitoring of Ambient Outdoor, Indoor Air Quality Pollutants, PM 10 and PM 2.5 Conducted to Evaluate Its Impact Analysis and Quantification in Industrial Area of Dammam, KSA
}

\author{
Sheeba Shafi', Bachir Yahia Khelif ${ }^{2}$ \\ ${ }^{1}$ Department of Nursing, College of Applied Medical Sciences, King Faisal University, Al-Ahsa, Saudi Arabia \\ ${ }^{2}$ Department of Public Health, College of Applied Medical Sciences, King Faisal University, Al-Ahsa, Saudi Arabia \\ Email: sheeba@kfu.edu.sa, bkhelif@kfu.edu.sa
}

How to cite this paper: Shafi, S., \& Khelif, B. Y. (2021). Environmental Monitoring of Ambient Outdoor, Indoor Air Quality Pollutants, PM 10 and PM 2.5 Conducted to Evaluate Its Impact Analysis and Quantification in Industrial Area of Dammam, KSA. Journal of Geoscience and Environment Protection, 9, 100-114. https://doi.org/10.4236/gep.2021.97007

Received: January 11, 2021

Accepted: July 25, 2021

Published: July 28, 2021

Copyright $\odot 2021$ by author(s) and Scientific Research Publishing Inc. This work is licensed under the Creative Commons Attribution International License (CC BY 4.0).

http://creativecommons.org/licenses/by/4.0/ (c) (i) Open Access

\begin{abstract}
The core objective of this study is to conduct precise and real-time monitoring of the industrial area Dammam located in the Kingdom of Saudi Arabia (KSA) which consists of a number of various chemicals, minerals and petrochemicals along with administrative blocks. The objective of this study is to monitor possible outdoor and indoor air pollutant in the industry working environment. This study shall help us to enable decision to be made on appropriate control measures as may be required to protect the health of employee and occupant who may be exposed to air contamination at workplace. Air pollution monitoring in ambient air environment finding shall be compared with national ambient air quality environmental standard while parameters monitoring in indoor air environment is compared with international standards such as occupational health and safety administration (OSHA), National institute for occupational safety and health (NIOSH) and Australian national health and medical research council (NHMRC). This environment review study for ensuring industry regulatory compliance for the facility and general authority of meteorology and environmental protection (GAMEP) previously known as presidency of meteorology environment (PME). This study shall be comprehensive in nature and cover two major types of monitoring and assessment such as ambient air quality monitoring and indoor air quality monitoring.
\end{abstract}

\section{Keywords}

Environmental Analysis, Presidency of Meteorology Environment, General 
Authority of Meteorology and Environmental Protection, Occupational Health and Safety Administration, National Institute for Occupational Safety and Health

\section{Introduction}

This work aims to conduct and identify level of ambient and indoor air quality pollutants targeted to the $3^{\text {rd }}$ industrial area of Dammam, KSA. This study and analysis help us to understand human working atmosphere in the industrial area. We have targeted critical and non-critical parameters to provide safe and conducive work atmosphere. Various environmental regulatory agencies ensure compliance of standard. Air pollution is an important public health problem in developed and developing nations (Issever et al., 2005). Worldwide, there are more than 2.7 million deaths due to air pollution (Nguyen \& Kim, 2006a, 2006b). Sources of air pollution include road traffic emissions, industrial emissions and domestic heating, or secondary formation pollutants (Marc-Andre et al., 2007; Kassomenos et al., 2006; Ogrin, 2007, Potoglou \& Kanaroglou, 2005) and (Charles et al., 2005). Common air pollutants that draw intense concerns include particulate matter $(\mathrm{PM})$, ozone $\left(\mathrm{O}_{3}\right)$, carbon monoxide $(\mathrm{CO})$, sulfur dioxide $\left(\mathrm{SO}_{2}\right)$, nitrogen dioxide $\left(\mathrm{NO}_{2}\right)$, and volatile organic compounds (VOCs) (Muir et al., 2006).

PM is a mixture of constituents that are formed by a large range of mechanisms associated with both natural and anthropogenic origins (Guerra et al., 1995). Exposure to high concentrations of PM increases the risk of lung cancer, respiratory diseases, arteriosclerosis, as well as changes in heart rate variability (Alves et al., 2000). In addition, aerosol particles have an impact on the environment in areas such as visibility and staining of buildings (de Kok et al., 2006; Sun et al., 2004; Virtanen et al., 2006).

$\mathrm{CO}$ is a flammable and poisonous gas emitted from incomplete combustion of carbonaceous material. Once absorbed, $\mathrm{CO}$ diffuses to plasma, passes across the red blood cell membrane, and finally enters the red blood cell cytoplasm where it binds to hemoglobin $(\mathrm{Hb})$ forming carboxyhemoglobin $(\mathrm{COHb})$. The affinity of $\mathrm{Hb}$ for $\mathrm{CO}$ is $210-300$ times greater than for oxygen, and $\mathrm{Hb}$ is thus incapable of combining with oxygen (Fang et al., 2006; Menut, 2003).

$\mathrm{NO}_{2}$ is released into the atmosphere from natural and man-made sources (e.g., burning of fossil fuels, construction, and mining). It is considered one of the main traffic-related air pollutants. A great number of studies have assessed the air pollution effects of nitrogen oxides $\left(\mathrm{NO}_{\mathrm{x}}\right)$ on human health. For instance, a cohort study reported significant increases in cardiopulmonary mortality for those living near major road areas in the Netherlands (Bono et al., 2007). $\mathrm{NO}_{\mathrm{x}}$ together with VOCs are responsible for the formation of photochemical smog and ground-level $\mathrm{O}_{3}$ in the presence of sunlight (Muir et al., 2006).

$\mathrm{SO}_{2}$ is emitted into the atmosphere mainly from anthropogenic sources such 
as the combustion of sulfur-containing fossil fuels (e.g., coal, oil, and natural gas). Peak levels of $\mathrm{SO}_{2}$ in the air can cause temporary breathing difficulty for people with asthma who are active outdoors. Longer-term exposures to high levels of $\mathrm{SO}_{2}$ and particles cause respiratory illness and aggravate existing heart disease. $\mathrm{SO}_{2}$ and $\mathrm{NO}_{\mathrm{x}}$ in the atmosphere are the major contributors to acidification. Acid rain damages forests and crops, changes the makeup of soil, and makes lakes and streams acidic and unsuitable for fish. VOCs are those organic compounds that have boiling points roughly in the range of $50^{\circ} \mathrm{C}-250^{\circ} \mathrm{C}$ (Shaw et al., 2005). Outdoors VOC concentrations are affected by season and temperature, proximity to emission sources such as industry, traffic and gas stations (Jia et al., 2008; Kwon et al., 2006; Kingdom of Saudi Arabia, Royal Commission for Jubail and Yanbu, 2010). Indoors VOCs concentrations are affected by outdoor levels due to the exchange of indoor and outdoor air, and by the numerous sources present indoors (Sapkota et al., 2006; Batterman et al., 2007; Charles et al., 2007; Tipton \& Dzombak, 2005).

The VOCs enter the atmosphere at room temperature and may cause headaches, dizziness, nausea and irritation of mucous membranes (Teixeira et al., 2009; Nathanson, 1995; U.S. Environmental Protection Agency, 2001; Sivertsen, 1997).

The air pollution problem inside or over the industrial area has different sources, including the plant operation and related activities. The general objective of this study is the assessment of air quality level inside the industrial area. This study will be considered as a benchmark of the environmental database for all industrial areas in the eastern province of the Kingdom of Saudi Arabia.

\section{Data and Research Methods}

\subsection{Research Area Overview and Data}

$3^{\text {rd }}$ Industrial City is located at 150 kilometers from city of Dammam which is a coastal city located in the Eastern Province of the Kingdom of Saudi Arabia (Figure 1). The geographic position system (GPS) coordinate of each corner of project site NW: $25^{\circ} 55^{\prime} 8.25^{\prime \prime} \mathrm{N}, 4^{\circ} 57^{\prime} 32.26^{\prime \prime} \mathrm{E}$; NE: $25^{\circ} 55^{\prime} 8.66^{\prime \prime} \mathrm{N}, 4^{\circ} 57^{\prime} 35.63^{\prime \prime} \mathrm{E}$; SE: $25^{\circ} 55^{\prime} 3.22^{\prime \prime} \mathrm{N}, 4^{\circ} 57^{\prime} 35.45^{\prime \prime E}$; and SW: $25^{\circ} 55^{\prime} 3.17^{\prime \prime} \mathrm{N}, 4^{\circ} 57^{\prime} 32.21^{\prime \prime E}$. During the present study, levels of the ambient air pollution were studied at two different locations. Location were the main gate and inside the premises. Ambient outdoor and indoor air quality pollutants include temperature, relative humidity, nitrogen dioxide, sulphur dioxide, hydrogen sulphide, ammonia, carbon monoxide, nitrogen oxide, ozone, carbon dioxide and total volatile organic compounds, particulate matter PM 10 and PM 2.5) were monitored at the boundary and inside of the facility. All reported values are in compliance with national ambient quality standards at all locations.

\subsection{Monitoring Methods}

A valid certified calibrated portable YES Plus LGA air quality monitor and AEROCET 531, particle mass monito (Class 1 laser product complies with IEC 
60825-1 Ed.1.1, EN60825-1 W/A11 and US 21 CFR 1040.10) were used to monitor ambient (outdoor) and indoor air quality. Air quality parameters monitored for ambient and indoor environment is shown in Table 1. Each location was monitored for fifteen (15) minutes. These monitoring were conducted at a height of $1.5 \mathrm{~m}$ above ground level. The height is approximately equal to the employee's breathing zone so that the concentrations measured will accurately reflect the concentration that is inhaled.

Air quality monitoring was conducted on December 14, 2019. Air quality monitoring data recorded at the various location of plant and various area mentioned in Table 2 of the facility is shown in Table 3.

\subsection{Temperature and Relative Humidity}

The ambient average temperature and relative humidity vary from $24^{\circ} \mathrm{C}-27.1^{\circ} \mathrm{C}$ and $18.4 \%$ - 26.2\% during the day time. Figure 2 and Figure 3 graphically illustrates the temperature and relative humidity data in respectively. The temperature and humidity potential to increase in the summer season due to desert natural environment.

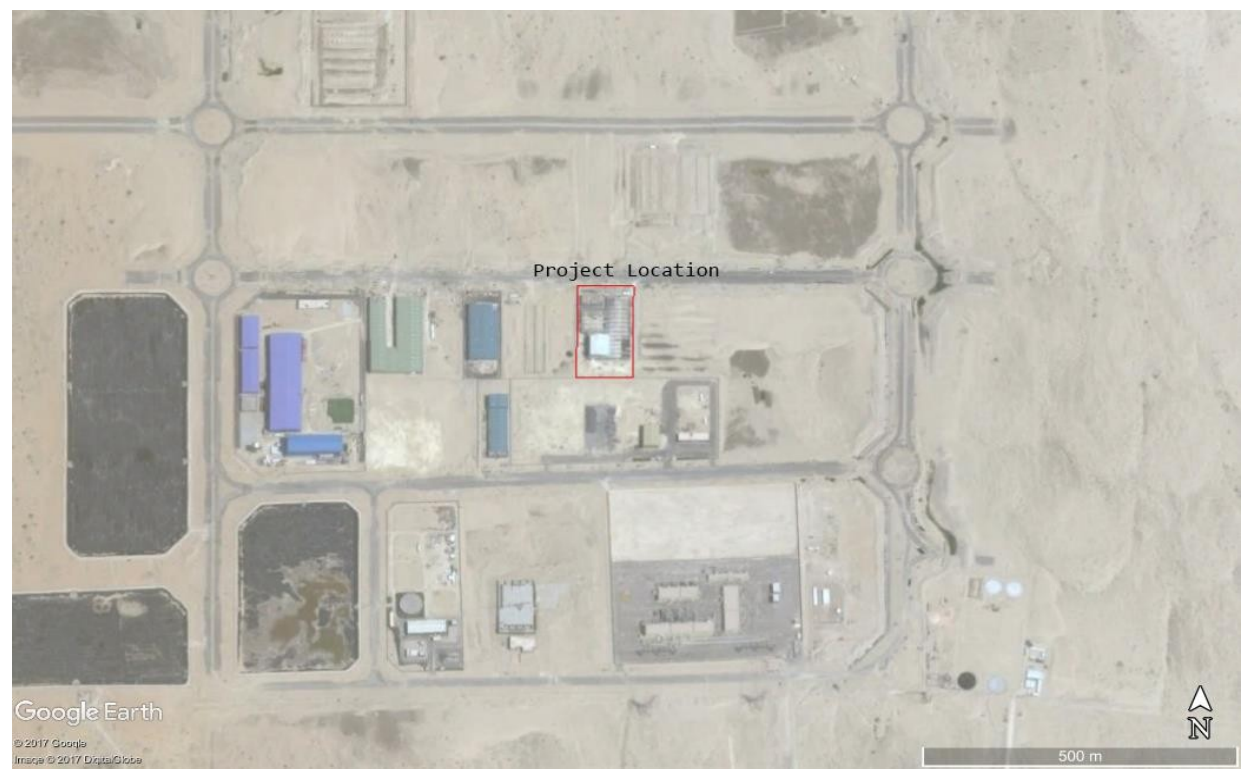

Figure 1. Facility location in industrial city (local context).

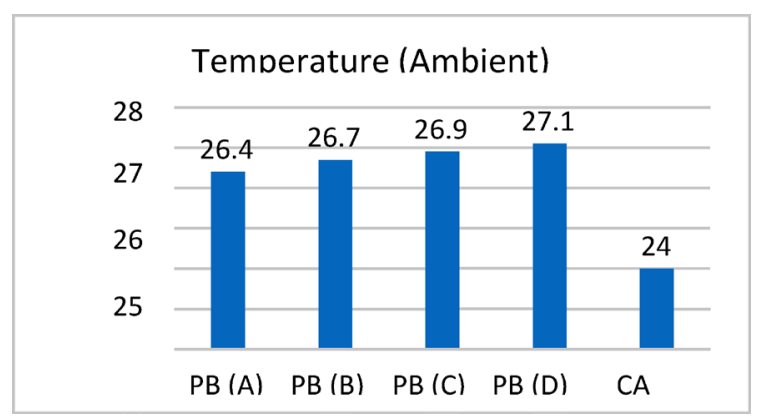

Figure 2. Ambient temperature data. 


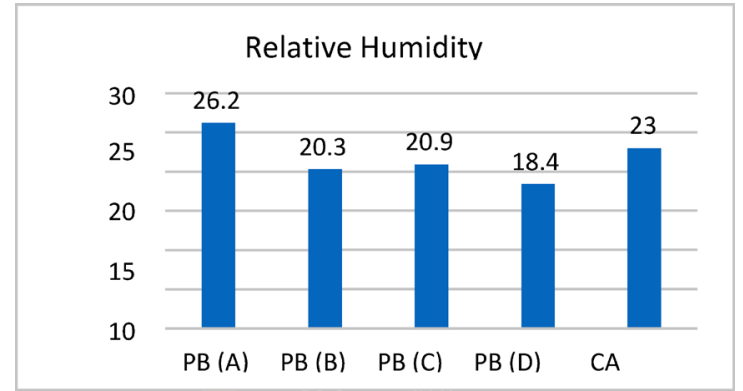

Figure 3. Ambient relative humidity data.

Table 1. Ambient and indoor air quality parameters.

\begin{tabular}{ll}
\hline Ambient air quality parameter & Indoor air quality parameter \\
\hline - Temperature & - Temperature \\
- Relative Humidity & - Relative Humidity \\
- Carbon monoxide $(\mathrm{CO})$ & - Carbon dioxide $\left(\mathrm{CO}_{2}\right)$ \\
- Nitrogen dioxide $\left(\mathrm{NO}_{2}\right)$ & - Carbon monoxide $(\mathrm{CO})$ \\
- Ozone $\left(\mathrm{O}_{3}\right)$ & - Nitrogen dioxide $\left(\mathrm{NO}_{2}\right)$ \\
- Hydrogen sulphide $\left(\mathrm{H}_{2} \mathrm{~S}\right)$ & - Nitric oxide $(\mathrm{NO})$ \\
- Sulphur dioxide $\left(\mathrm{SO}_{2}\right)$ & - Ozone $\left(\mathrm{O}_{3}\right)$ \\
- Particulate Matter $(\mathrm{PM} \mathrm{10)}$ & - Ammonia $\left(\mathrm{NH} \mathrm{H}_{3}\right)$ \\
- Particulate Matter $(\mathrm{PM} 2.5)$ & - Hydrogen sulphide $\left(\mathrm{H}_{2} \mathrm{~S}\right)$ \\
& - Sulphur dioxide $\left(\mathrm{SO}_{2}\right)$ \\
& - Total Volatile organic compounds (TVOCs) \\
& - Particulate Matter $(\mathrm{PM} \mathrm{10)}$ \\
& - Particulate Matter $(\mathrm{PM} \mathrm{2.5)}$ \\
& - Total Suspended Particulate \\
\hline
\end{tabular}

Table 2. Ambient and indoor monitoring locations.

\begin{tabular}{ll}
\hline Ambient Monitoring Locations & Indoor Monitoring location \\
\hline - Plant boundary A-PB (A) & - Laboratory-Lab \\
- Plant boundary B-PB (B) & - Production Area 1-PA 1 \\
- Plant boundary C-PB (C) & - Production Area 2-PA 2 \\
- Plant boundary D-PB (D) & - Warehouse 1-WH 1 \\
- Crushing Area (CA) & - Warehouse 2-WH 2 \\
\hline
\end{tabular}

Table 3. Ambient air quality monitoring data.

\begin{tabular}{|c|c|c|c|c|c|c|c|c|c|}
\hline Location & $\begin{array}{c}\text { Temp. } \\
{ }^{\circ} \mathrm{C}\end{array}$ & $\begin{array}{c}\text { RH } \\
\%\end{array}$ & $\begin{array}{l}\mathrm{NO}_{2} \\
\mathrm{ppm}\end{array}$ & $\begin{array}{l}\mathrm{SO}_{2} \\
\mathrm{ppm}\end{array}$ & $\begin{array}{l}\mathrm{H}_{2} \mathrm{~S} \\
\mathrm{ppm}\end{array}$ & $\begin{array}{c}\mathrm{CO} \\
\mathrm{ppm}\end{array}$ & $\begin{array}{c}\mathrm{O}_{3} \\
\mathrm{ppm}\end{array}$ & $\begin{array}{c}\text { PM } 2.5 \\
\mu g / m^{3}\end{array}$ & $\begin{array}{l}\mathrm{PM} 10 \\
\mu \mathrm{g} / \mathrm{m}^{3}\end{array}$ \\
\hline Plant Boundary (PB-A) & 26.4 & 26.2 & 0.0 & 0.5 & 0.04 & 0.9 & 0.06 & 29.2 & 159.3 \\
\hline Plant Boundary (PB-B) & 26.7 & 20.3 & 0.0 & 0.0 & 0.03 & 1.7 & 0.09 & 27.8 & 156.7 \\
\hline Plant Boundary (PA-C) & 26.9 & 20.9 & 0.0 & 0.0 & 0.04 & 2.0 & 0.09 & 21.7 & 103.3 \\
\hline Plant Boundary (PA-D) & 27.1 & 18.4 & 0.0 & 0.1 & 0.04 & 2.1 & 0.08 & 18.3 & 89.2 \\
\hline Crushing Area (CA) & 24.0 & 23.0 & 0.0 & 0.0 & 0.03 & 1.0 & 0.08 & 25.2 & 157.9 \\
\hline
\end{tabular}




\subsection{Ambient Air Pollutant}

Reported data of sulphur dioxide $\left(\mathrm{SO}_{2}\right)$, carbon monoxide $(\mathrm{CO})$, ozone $\left(\mathrm{O}_{3}\right)$, and hydrogen sulfide $\left(\mathrm{H}_{2} \mathrm{~S}\right)$ and particulate matter (PM 10 and PM 2.5) are shown in Figures 4-9 respectively. All values are in compliance with national ambient quality standards at all locations. Figures 7-9 depict PM 2.5, 10 and hydrogen sulphide.

\section{Indoor Air Quality Environment}

Air quality parameters include temperature, relative humidity, nitrogen dioxide, sulphur dioxide, hydrogen sulphide, ammonia, carbon monoxide, nitrogen oxide, ozone, carbon dioxide and total volatile organic compounds, particulate matter PM 10, particulate matter PM 2.5 and total suspended particulate (TSP). Indoor air quality recorded at the facility is shown in Table 4.

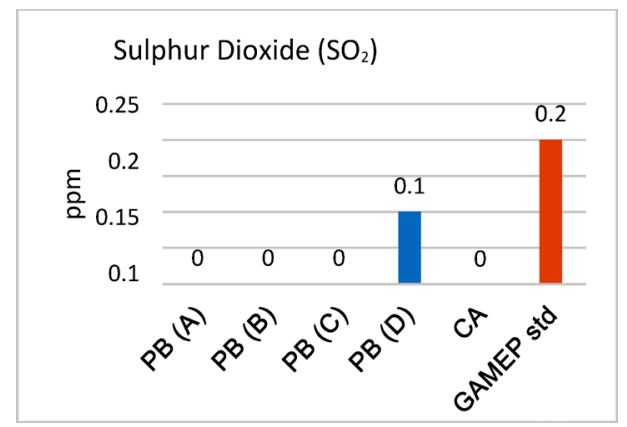

Figure 4. Ambient sulfur dioxide data.

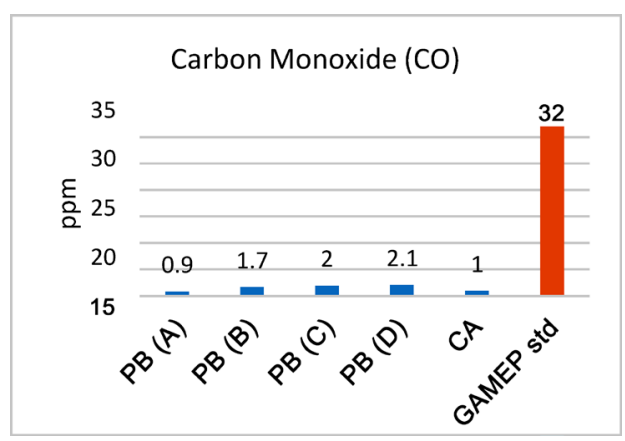

Figure 5. Ambient carbon monoxide data.

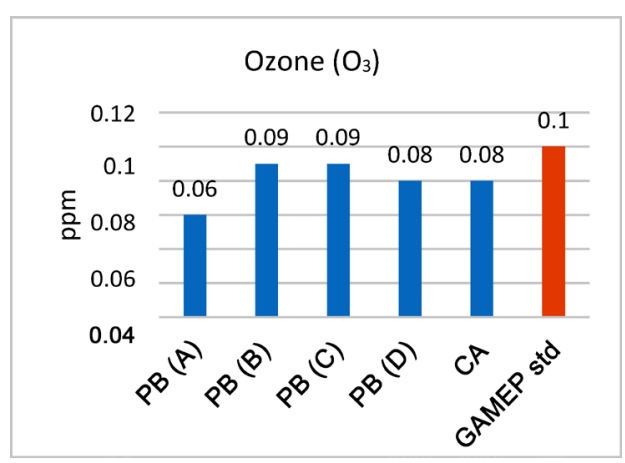

Figure 6. Ambient ozone data. 


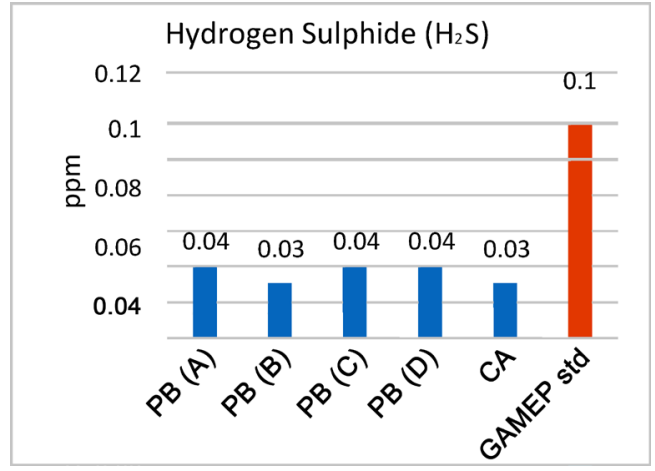

Figure 7. Ambient hydrogen sulphide data.

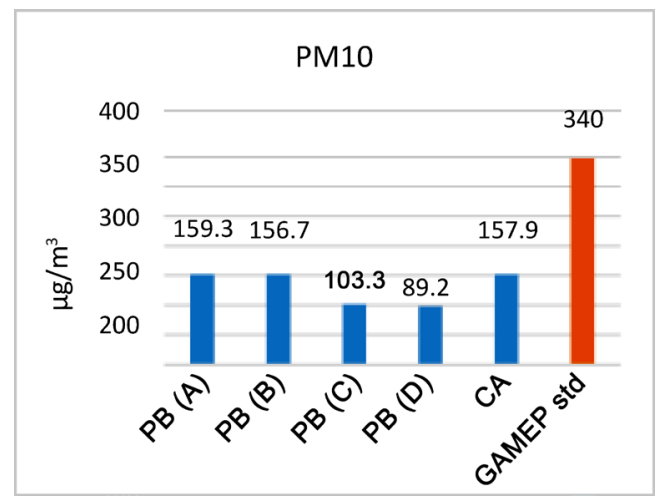

Figure 8. Ambient PM 10 data.

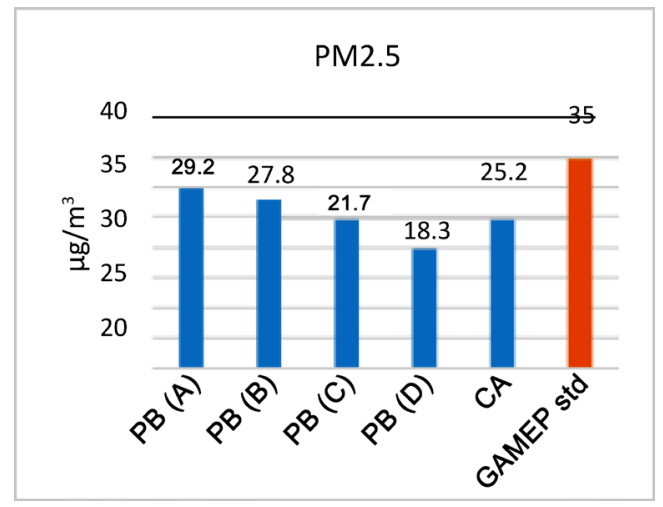

Figure 9. Ambient PM 2.5 data.

Table 4. Indoor air quality monitoring data.

\begin{tabular}{cccccccccccccccc}
\hline Location & $\begin{array}{c}\mathrm{Temp} . \\
{ }^{\circ} \mathrm{C}\end{array}$ & $\begin{array}{c}\mathrm{RH} \\
\%\end{array}$ & $\begin{array}{c}\mathrm{NO}_{2} \\
\mathrm{ppm}\end{array}$ & $\begin{array}{c}\mathrm{SO}_{2} \\
\mathrm{ppm}\end{array}$ & $\begin{array}{c}\mathrm{H}_{2} \mathrm{~S} \\
\mathrm{ppm}\end{array}$ & $\begin{array}{c}\mathrm{NH}_{3} \\
\mathrm{ppm}\end{array}$ & $\begin{array}{c}\mathrm{CO} \\
\mathrm{ppm}\end{array}$ & $\begin{array}{c}\mathrm{NO} \\
\mathrm{ppm}\end{array}$ & $\begin{array}{c}\mathrm{O}_{3} \\
\mathrm{ppm}\end{array}$ & $\begin{array}{c}\mathrm{CO}_{2} \\
\mathrm{ppm}\end{array}$ & $\begin{array}{c}\mathrm{VOC} \\
\mathrm{mg} / \mathrm{m}^{3}\end{array}$ & $\begin{array}{c}\mathrm{PM} 2.5 \\
\mu \mathrm{g} / \mathrm{m}^{3}\end{array}$ & $\begin{array}{c}\mathrm{PM} 10 \\
\mu \mathrm{g} / \mathrm{m}^{3}\end{array}$ & $\begin{array}{c}\mathrm{TSP} \\
\mu \mathrm{g} / \mathrm{m}^{3}\end{array}$ \\
\hline Lab & 22.1 & 31.3 & 0.0 & 0.1 & 0.4 & 0.0 & 2.1 & 0.7 & 0.0 & 406.3 & 234.0 & 30.2 & 67.3 & 73.8 \\
PA 1 & 23.7 & 23.0 & 0.0 & 0.6 & 0.3 & 0.0 & 2.5 & 0.3 & 0.3 & 361.4 & 310.0 & 118.3 & 195.2 & 957.8 \\
PA 2 & 23.3 & 24.0 & 0.0 & 0.0 & 0.3 & 0.0 & 0.9 & 0.3 & 0.7 & 338.5 & 334.0 & 70.5 & 130.4 & 810.2 \\
WH 1 & 23.4 & 23.2 & 0.0 & 0.0 & 0.3 & 1.0 & 1.0 & 0.4 & 0.6 & 338.8 & 189.0 & 61.5 & 145.3 & 557.5 \\
WH 2 & 23.4 & 24.0 & 0.0 & 0.0 & 0.3 & 1.0 & 1.0 & 0.4 & 0.4 & 367.7 & 264.0 & 50.8 & 105.7 & 390.9 \\
\hline
\end{tabular}




\subsection{Temperature and Relative Humidity}

Temperature and relative humidity are often referred to as comfort parameters and contribute to the thermal comfort for indoor working personnel. There are no "ideal" humidity and temperature standards suitable for all building occupants or indoor work areas therefore different organizations have varying standards. The average temperature and relative humidity data monitored at each location is compared with NIOSH standards.

Figure 10 and Figure 11 graphically illustrates the temperature and relative humidity data with NIOSH lower and upper limit standard. Temperature and relative humidity were in compliance with NIOSH standards. The monitoring was undertaken in the winter season but there is potential to increase temperature and humidity in the summer season.

\subsection{Indoor Air Pollutants}

Reported data of carbon monoxide (CO), sulphur dioxide $\left(\mathrm{SO}_{2}\right)$, hydrogen sulfide $\left(\mathrm{H}_{2} \mathrm{~S}\right)$, nitric oxide $(\mathrm{NO})$, ozone $\left(\mathrm{O}_{3}\right)$, total volatile organic compounds (TVOCs) and carbon dioxide $\left(\mathrm{CO}_{2}\right)$, particulate matter (PM 10), particulate matter (PM 2.5) and total suspended particulates with standards are shown in Figures 12-21 respectively. Figure 19 and Figure 20 represent the PM 2.5 and PM 10 data was reported above IDPH $\left(65 \mu \mathrm{g} / \mathrm{m}^{3}\right.$ and $\left.150 \mu \mathrm{g} / \mathrm{m}^{3}\right)$ standards.

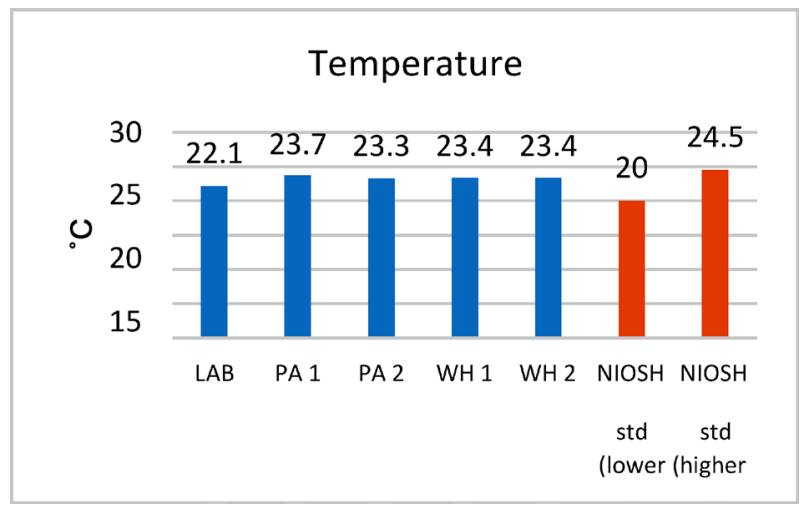

Figure 10. Indoor temperature data.

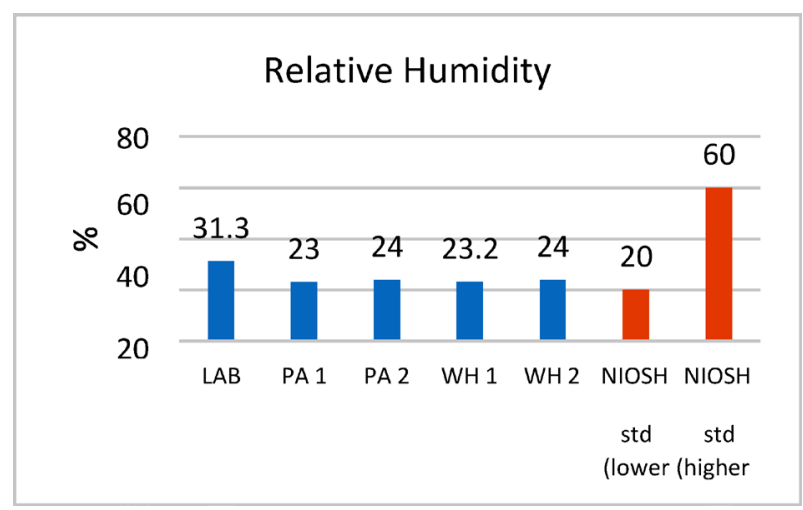

Figure 11. Indoor relative humidity. 


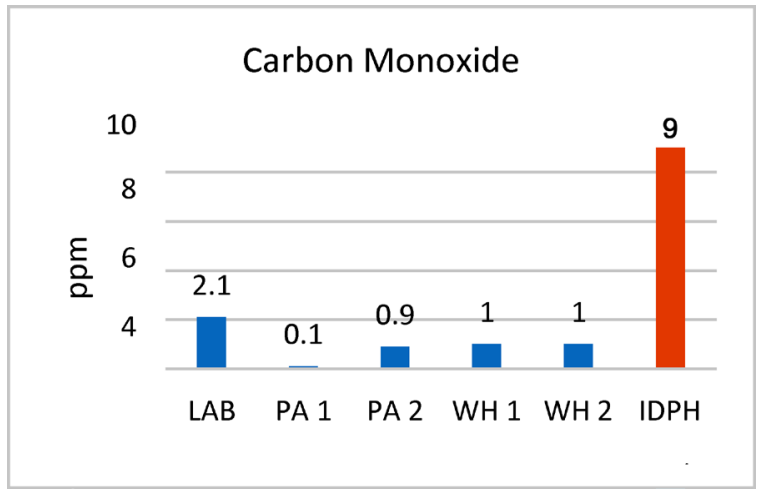

Figure 12. Indoor carbon monoxide data.

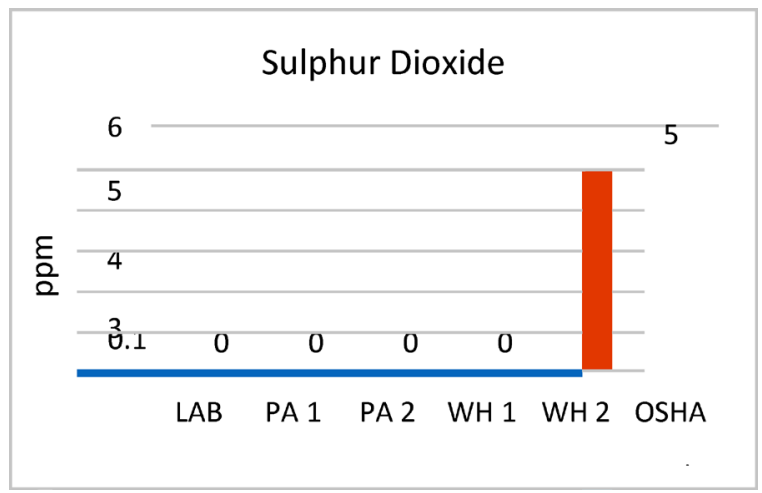

Figure 13. Indoor sulfur dioxide data.

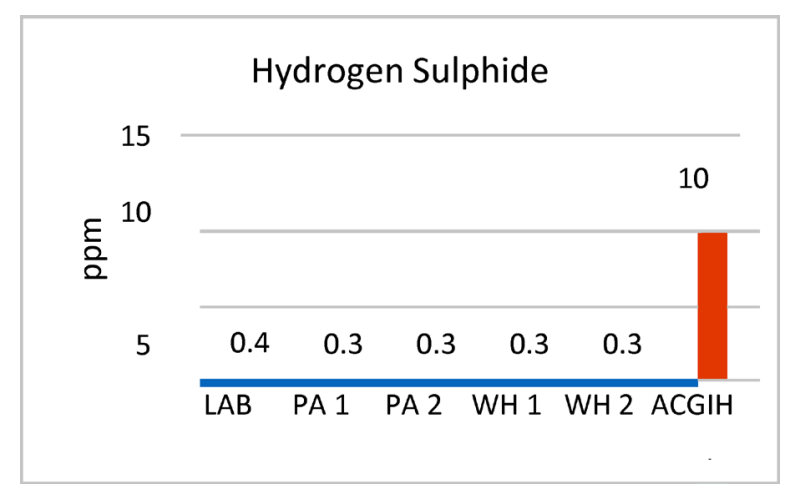

Figure 14. Indoor hydrogen sulfide data.

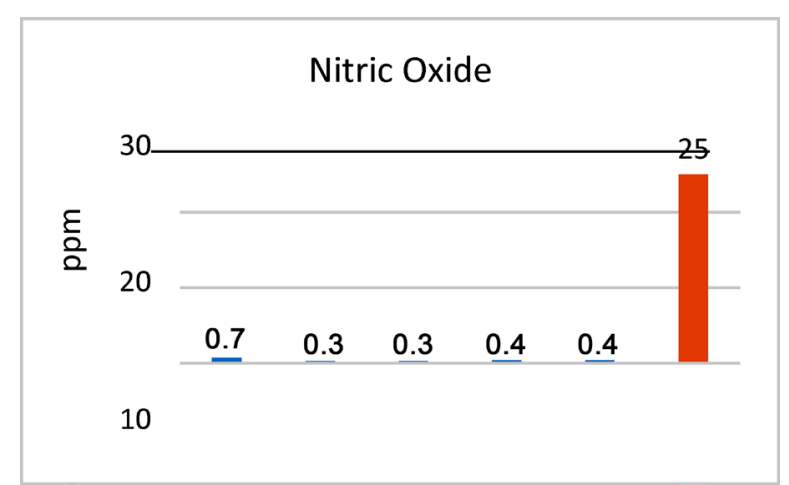

Figure 15. Indoor nitric oxide data. 


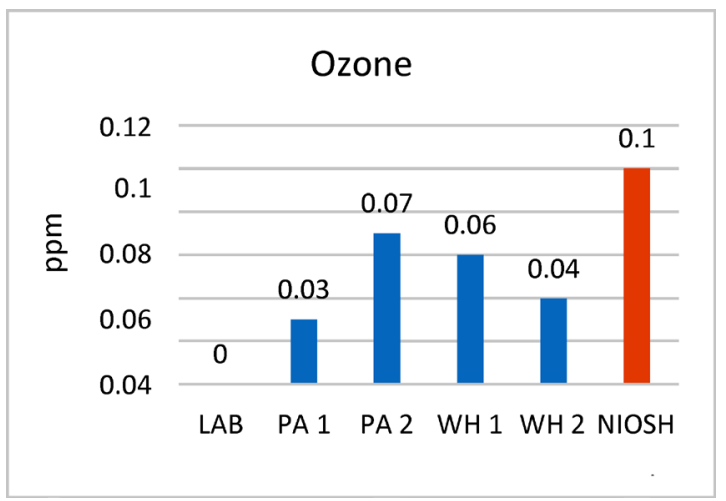

Figure 16. Indoor ozone data.

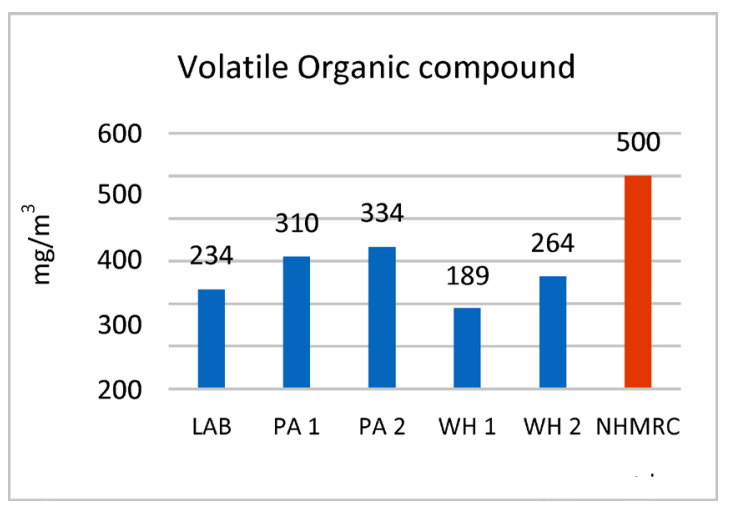

Figure 17. Indoor VOC data.

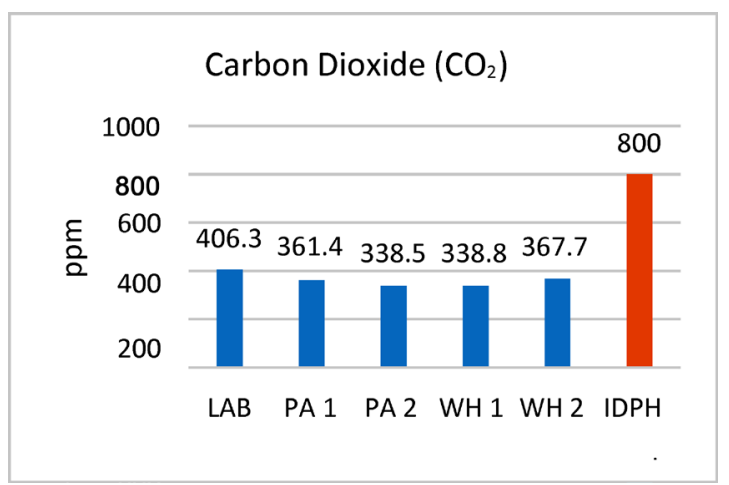

Figure 18. Indoor carbon dioxide data.

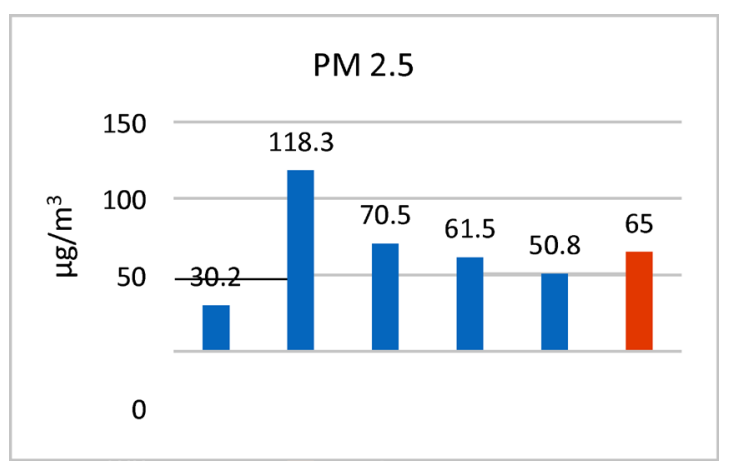

Figure 19. Indoor PM 2.5 data. 


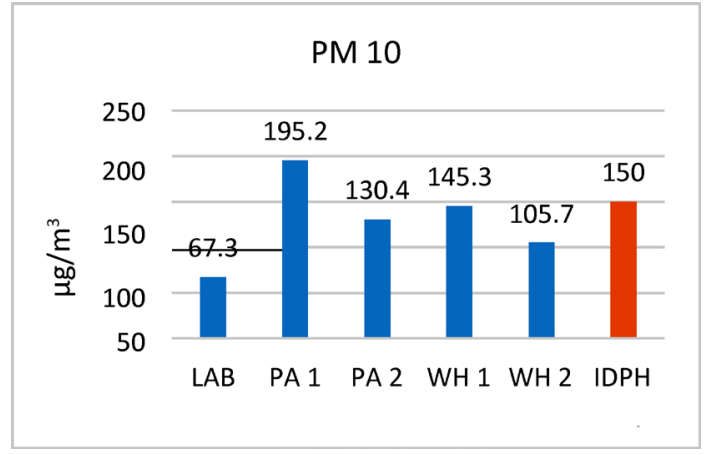

Figure 20. Indoor PM 10 data.

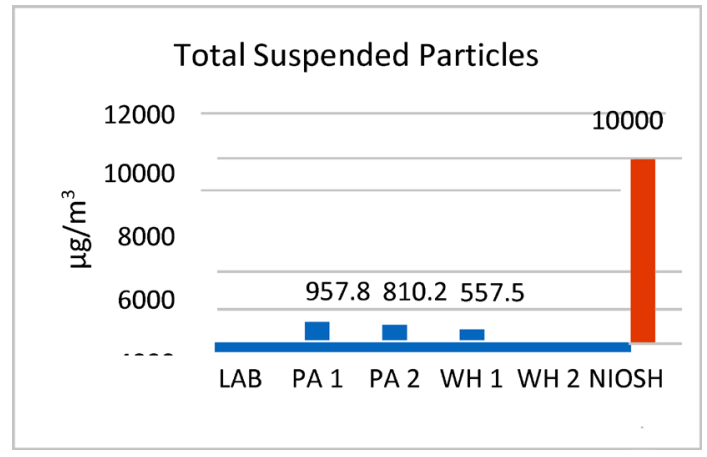

Figure 21. Indoor total suspended particles data.

Table 5. Ambient air quality standards.

\begin{tabular}{|c|c|c|c|}
\hline Parameter & Time-weighted average $\mu \mathrm{g} / \mathrm{Nm}^{3}$ (ppm) & Averaging time & Number of allowable exceedances \\
\hline \multirow{2}{*}{ Carbon Monoxide } & $10,000(8.1)$ & 8 hours & \multirow{2}{*}{ None } \\
\hline & $40,000(32)$ & 1 hour & \\
\hline Lead & $0.5(0.00005)$ & Annual & N/A \\
\hline \multirow{2}{*}{ Nitrogen Dioxide } & $660(0.35)$ & 1 hour & 2 times per 30 days \\
\hline & $100(0.05)$ & Annual & N/A \\
\hline \multirow{3}{*}{ Sulphur Dioxide } & $730(0.28)$ & 1 hour & 2 times per annum \\
\hline & $365(0.14)$ & 24 hours & 1 time per annum \\
\hline & $80(0.03)$ & Annual & N/A \\
\hline Benzene & $5(0.0015)$ & Annual & N/A \\
\hline \multirow{2}{*}{ Particulate Matter (PM 10) } & 340 (variable) & 24 hours & 24 times per annum ${ }^{\mathrm{b}}$ \\
\hline & 80 (variable) & Annual & N/A \\
\hline \multirow{2}{*}{ Particulate Matter (PM 2.5) } & 35 & 24 hours & 24 times per annum $^{c}$ \\
\hline & 15 & Annual & N/A \\
\hline \multirow{2}{*}{ Ozone } & $235(0.12)$ & 1 hour & 2 times per 30 days \\
\hline & $157(0.08)$ & 8 hours & 2 times per 7 days \\
\hline \multirow{2}{*}{ Hydrogen Sulphide } & $150(0.1)$ & 24 hours & 10 times per annum \\
\hline & $40(0.03)$ & Annual & N/A \\
\hline
\end{tabular}

${ }^{a}$ Violations will only be reportable where validated data is available for $98 \%$ of measurements; ${ }^{\text {b }}$ The average $90^{\text {th }}$ Percentile 24 -hour concentration must not exceed $340 \mu \mathrm{g} / \mathrm{Nm}^{3}$; ${ }^{\text {c }}$ The average $90^{\text {th }}$ Percentile 24-hour concentration must not exceed $35 \mu \mathrm{g} / \mathrm{Nm}^{3}$. 
Table 6. Indoor air quality standards.

\begin{tabular}{|c|c|c|c|c|c|}
\hline Parameter & IDPH & NHMRC & NIOSH & OSHA & ACGIH \\
\hline Relative humidity & $20 \%-60 \%$ & - & $20 \%-60 \%$ & $20 \%-60 \%$ & - \\
\hline \multirow{3}{*}{ Temperature $\left({ }^{\circ} \mathrm{C}\right)$} & $20-24$ (winter) & \multirow{3}{*}{$20-26$} & \multirow{3}{*}{$20-24.5$} & \multirow{3}{*}{$20-26$} & - \\
\hline & & & & & \\
\hline & 22.8 - 26.1 (summer) & & & & \\
\hline Carbon dioxide & $\begin{array}{c}1000 \text { ppm } \\
800 \text { ppm preferred }\end{array}$ & - & 1000 & $5000 \mathrm{ppm}$ & $5000 \mathrm{ppm}$ \\
\hline Carbon monoxide & $9 \mathrm{ppm}$ & 9 ppm (8 hours) & $35 \mathrm{ppm}$ & $50 \mathrm{ppm}$ & $25 \mathrm{ppm}$ \\
\hline $\begin{array}{l}\text { Volatile organic } \\
\text { compounds }\end{array}$ & - & $\begin{array}{c}500 \mu \mathrm{g} / \mathrm{m}^{3} \\
(0.218 \mathrm{ppm}) \\
(1 \text { hour })\end{array}$ & - & & \\
\hline Sulfur dioxide & - & $200 \mathrm{ppb}$ (1 hour) & $\begin{array}{c}2 \mathrm{ppm} \\
(5 \mathrm{ppm} / 15 \text { minute })\end{array}$ & $5 \mathrm{ppm}(8 \mathrm{hr})$ & $5 \mathrm{ppm}(15 \mathrm{~min})$ \\
\hline Hydrogen sulfide & $0.01 \mathrm{ppm}$ & - & - & $20 \mathrm{ppm}$ & $10 \mathrm{ppm}$ \\
\hline Ozone & $0.08 \mathrm{ppm}$ & & $0.1 \mathrm{ppm}$ & $0.1 \mathrm{ppm}$ & $0.05 \mathrm{ppm}$ \\
\hline Nitrogen dioxide & $\begin{array}{c}0.05 \text { ppm } \\
\text { (24 hours average) }\end{array}$ & - & $1 \mathrm{ppm} / 15 \mathrm{~min}$ & $5 \mathrm{ppm}(8 \mathrm{hr})$ & $\begin{array}{c}3 \text { ppm } \\
5 \text { ppm (15 min) }\end{array}$ \\
\hline Nitric Oxide & - & - & - & $25 \mathrm{ppm}$ & - \\
\hline Ammonia & - & - & - & $50 \mathrm{ppm}$ & - \\
\hline PM 10 & $150 \mu \mathrm{g} / \mathrm{m}^{3}$ & - & - & - & - \\
\hline PM 2.5 & $65 \mu \mathrm{g} / \mathrm{m}^{3}$ & $5000 \mu \mathrm{g} / \mathrm{m}^{3}$ & $3000 \mu \mathrm{g} / \mathrm{m}^{3}$ & - & - \\
\hline TSP & - & $15,000 \mu \mathrm{g} / \mathrm{m}^{3}$ & $10,000 \mu \mathrm{g} / \mathrm{m}^{3}$ & - & - \\
\hline
\end{tabular}

All values are in compliance with OSHA/NIOSH/ACGIH/IDPH/NHMRC standards where applicable except particulate matter PM 10 and PM 2.5 at production area.

\subsection{Ambient Air Quality Standard}

Ambient air quality standards (AAQS) of kingdom of Saudi Arabia are outlined in Table 5. There are different agencies globally that develops Indoor air quality standards. The indoor air quality standards from international agencies from the National Institute of Occupational Health and Safety (NIOSH), Occupational Safety and Health Administration (OSHA), American Conference of Governmental Industrial Hygienists (ACGIH), Illinois Department of Public Health (IDPH), Australian National Health and Medical Research Council (NHMRC) are represented in Table 6. These standards will be used to evaluate monitoring data where applicable.

\section{Conclusion}

The findings of the ambient air quality monitoring study show that the air quality parameters are within the acceptable limit of national ambient air quality standards. The findings of the indoor air quality monitoring study show that the 
air quality parameters are within the acceptable limit of working environment except for particulate matter, PM 10 and PM 2.5 at production area.

We recommend wearing appropriate personal protective equipment (PPE) and a nose mask must be mandatory at production areas.

\section{Conflicts of Interest}

The authors declare no conflicts of interest regarding the publication of this paper.

\section{References}

Alves, C. A., Pio, C. A., \& Duarte, A. C. (2000). Particle Size Distributed Organic Compound in a Forest Atmosphere. Environmental Science Technology, 25, 2113-2140.

Batterman, S., Jia, C., \& Hatzivailis, G. (2007). Migration of Volatile Organic Compounds from Attached Garages to Residences: A Major Exposure Source. Environmental Research, 104, 224-240. https://doi.org/10.1016/j.envres.2007.01.008

Bono, R., Piccioni, P., Traversi, D., Degan, R., Grosa, M., Bosello, G., Gilli, G., Arossa, W., \& Bugiani, M. (2007). Urban Air Quality and Carboxyhemoglobin Levels in a Group of Traffic Policemen. Science of the Total Environment, 376, 109-115. https://doi.org/10.1016/j.scitotenv.2007.01.086

Charles, K., Magee, R.J., Won, D., \& Lusztyk, E. (2005). Indoor Air Quality Guidelines and Standards. National Research Council Canada, RR-204.

Charles, S., Batterman, S., \& Jia, C. (2007). Composition and Emissions of VOCs in Mainand Side-Stream Smoke of Research Cigarettes. Atmospheric Environment, 41, 5371-5384. https://doi.org/10.1016/j.atmosenv.2007.02.020

de Kok, T. M. C., Driece, H. A. L., Hogervorst, J. G. F., \& Briede, J. J. B. (2006). Toxicological Assessment of Ambient and Traffic-Related Particulate Matter: A Review of Recent Studies. Mutation Research, 613, 103-122. https://doi.org/10.1016/j.mrrev.2006.07.001

Fang, G.-C., Wu, Y.-S., Chen, J.-C., Rau, J.-Y., Huang, S.-H., \& Lin, C.-K. (2006). Concentrations of Ambient Air Particulates (TSP, $\mathrm{PM}_{2.5}$ and $\mathrm{PM}_{2.5-10}$ ) and Ionic Species at Offshore Areas near Taiwan Strait. Journal of Hazardous Materials B, 132, 269-276. https://doi.org/10.1016/j.jhazmat.2005.09.049

Guerra, A. I., Lerda, D., \& Martines, C. (1995). Benzene Emissions from Motor Vehicle Traffic in the Urban Area of Milan: Hypothesis of Health Impact Assessment. Atmospheric Environment, 29, 3559-3569. https://doi.org/10.1016/1352-2310(95)00205-D

Issever, H., Disci, R., Hapcioglu, B., Vatansever, S., Karan, M., Akkaya, V., \& Erk, O. (2005). The Effect of Air Pollution and Meteorological Parameters in Istanbul on Hospital Admissions for Acute Coronary Syndrome. Indoor and Built Environment, 14, 157-164. https://doi.org/10.1177/1420326X05052798

Jia, C., Batterman, S., \& Godwin, C. (2008). VOCs in Industrial, Urban and Suburban Neighborhoods-Part 2: Factors Affecting Indoor and Outdoor Concentrations. Atmos pheric Environment, 42, 2101-2116. https://doi.org/10.1016/j.atmosenv.2007.11.047

Kassomenos, P., Karakitsios, S., \& Papaloukas, C. (2006). Estimation of Daily Traffic Emissions in a South-European Urban Agglomeration during a Workday. Evaluation of Several "What If" Scenarios. Science of the Total Environment, 370, 480-490. https://doi.org/10.1016/j.scitotenv.2006.08.018

Kingdom of Saudi Arabia, Royal Commission for Jubail and Yanbu (2010). Royal Com- 
mission Environmental Regulations, Volume 1.

Kwon, J., Weisel, C., Turpin, B. J., Zhang, J. F., Korn, L. R., Morandi, M. T., Stock, T. H., \& Colome, S. (2006). Source Proximity and Outdoor-Residential VOC Concentrations: Results from the RIOPA Study. Environmental Science and Technology, 40, 4074-4082. https://doi.org/10.1021/es051828u

Marc-Andre, R., Chimonasa, M. R., Bradford, D., \& Gessne, B. D. (2007). Airborne Particulate Matter from Primarily Geologic, Non-Industrial Sources at Levels below National Ambient Air Quality Standards Is Associated with Outpatient Visits for Asthma and Quick-Relief Medication Prescriptions among Children Less than 20 Years Old Enrolled in Medicaid in Anchorage, Alaska. Environmental Research, 103, 397-404. https://doi.org/10.1016/j.envres.2006.08.013

Menut, L. (2003). Adjoint Modeling for Atmospheric Pollution Process Sensitivity at Regional Scale. Journal of Geophysical Research, 108, 8562. https://doi.org/10.1029/2002JD002549

Muir, D., Longhurst, J. W. S., \& Tubb, A. (2006). Characterization and Quantification of the Sources of $\mathrm{PM}_{10}$ during Air Pollution Episodes in the UK. Science of the Total Environment, 358, 188-205. https://doi.org/10.1016/j.scitotenv.2005.04.019

Nathanson, T. (1995). Indoor Air Quality in Office Buildings: A Technical Guide. Minister of National Health and Welfare, Canada.

Nguyen, H. T., \& Kim, K.-H. (2006a). Comparison of Spatiotemporal Distribution Patterns of $\mathrm{NO}_{2}$ between Four Different Types of Air Quality Monitoring Stations. Chemosphere, 65, 201-212. https://doi.org/10.1016/j.chemosphere.2006.02.061

Nguyen, H. T., \& Kim, K.-H. (2006b). Evaluation of $\mathrm{SO}_{2}$ Pollution Levels between Four Different Types of Air Quality Monitoring Stations. Atmospheric Environment, 40, 7066-7081. https://doi.org/10.1016/j.atmosenv.2006.06.011

Ogrin, M. (2007). Air Pollution Due to Road Traffic in Ljubljana. Dela, 27, 199-214. https://doi.org/10.4312/dela.27.199-214

Potoglou, D., \& Kanaroglou, P. S. (2005). Carbon Monoxide Emissions from Passenger Vehicles: Predictive Mapping with an Application to Hamilton, Canada. Transportation Research Part D, 10, 97-109. https://doi.org/10.1016/j.trd.2004.11.003

Sapkota, B., Shrestha, M. L., Kaga, A., Kondo, A., \& Inoue, Y. (2006). Ground Level Ozone Concentrations and Its Association with $\mathrm{NO}_{\mathrm{x}}$ and Meteorological Parameters in Kathmandu Valley, Nepal. Atmospheric Environment, 40, 8081-8087. https://doi.org/10.1016/j.atmosenv.2006.07.011

Shaw, C. Y., Won, D., \& Reardon, J. (2005). Managing Volatile Organic Compounds and Indoor Air Quality in Office Buildings-An Engineering Approach. National Research Council Canada, RR-205.

Sivertsen, B. (1997). Air Quality Monitoring Systems and Applications. Norwegian Institute for Air Research, NIL TR 11/97, Q-303.

Sun, Y., Zhuang, G., Wang, Y., Han, L., Guo, J., Dan, M., Zhang, W., Wang, Z., \& Hao, Z. (2004). The Air-Borne Particulate Pollution in Beijing-Concentration, Composition, Distribution and Sources. Atmospheric Environment, 38, 5991-6004. https://doi.org/10.1016/j.atmosenv.2004.07.009

Teixeira, E. C., de Santana, E. R., Wiegand, F., \& Fachel, J. (2009). Measurement of Surface Ozone and Its Precursors in an Urban Area in South Brazil. Atmospheric Environment, 43, 2213-2220. https://doi.org/10.1016/j.atmosenv.2008.12.051

Tipton, K. J., \& Dzombak, D. A. (2005). Environmental Indicators for Carnegie Mellon University: Baseline Assessment 2004. 
U.S. Environmental Protection Agency (2001). Review of the National Ambient Air Quality Standards for Particulate Matter: Policy Assessment of Scientific and Technical Information. Office of Air Quality Planning and Standards.

Virtanen, A., Keskinen, J., Ristimaki, J., Ronkko, T., \& Vaaraslahti, K. (2006). Reducing Particulate Emissions in Traffic and Transport. Libris Oy. 